\title{
Metopic Suture Synostosis (Trigonocephaly): A Case Report
}

\author{
Ercan BAL ${ }^{1}$, İsmail BOZKURT², Cevat AKINCI ${ }^{2}$, Nebi YILMAZ ${ }^{1}$
}

${ }^{1}$ Department of Neurosurgery, Yıldırım Beyazıt University, Ankara, Turkiye.

${ }^{2}$ Department of Neurosurgery, Ankara Atatürk Training and Research Hospital, Ankara, Turkiye.

\section{ABSTRACT}

This study aimed to present the case of a patient with trigonocephaly who was operated at 6 months of age with bifrontal craniotomy and fronto-orbital advancement. Trigonocephaly is a premature fusion and ossification of the metopic suture. The calvarial growth restriction causes decreased intracranial volume. Thus, a surgical intervention is indicated to restore the volume of the skull along with appearance.

Key words: Craniosynostosis, fronto-orbital advancement, metopic synostosis, treatment, trigonocephaly

\section{INTRODUCTION}

Craniosynostosis is a premature fusion of single or multiple cranial sutures. It occurs in one of 2000-2500 live births $(1,2)$. Trigonocephaly is a form of craniosynostosis arising from the premature fusion and ossification of the metopic suture, which separates the two frontal bones and physiologically closes at 3-8 months of age $(3,4)$. Described first by Welcker in 1862, trigonocephaly is a Greek term meaning triangular forehead. Metopic synostosis occurs in between $1 / 700$ and 1/15.000 newborns $(5,6)$. This results in a skull with a triangular forehead and deficient lateral orbital rims add to the supraorbital retrusion and bitemporal indentations. The growth restriction results in decreased intracranial volume. Thus, a surgical intervention is indicated to restore the volume of the skull along with appearance (7). This study aimed to present the case of a child born with trigonocephaly who was operated at 6 months of age with bifrontal craniotomy and fronto-orbital advancement.

\section{CASE}

The 6-month-old patient was born as the fifth child to the mother. He had a breech presentation with his head caught in the birth canal during delivery. Ever since his birth the family noticed an abnormally shaped skull with hypotelorism (Figures 1a,b). They applied to a neurosurgery clinic where they were advised for surgery when the child reached 6 months of age. His cranial computerized tomography (CT) revealed a fused metopic suture (Figures 1c-f). Diagnosed with trigonocephaly he underwent surgery under general anesthesia. A zigzag skin incision was made from the anterior portion of one tragus to the other. The scalp was dissected up to both temporal sides and supraorbital region. The dissection was performed using frontozygomatic and frontonasal sutures $2 \mathrm{~cm}$ posterior of the orbital ceilings while preserving supraorbital nerves bilaterally. The temporal muscles were dissected up to squamous sutures. A bone drill was used to perform bifrontal craniotomy and bilateral supraorbital rim osteotomy. The dura mater was dissected from the floor of the anterior fossa. 

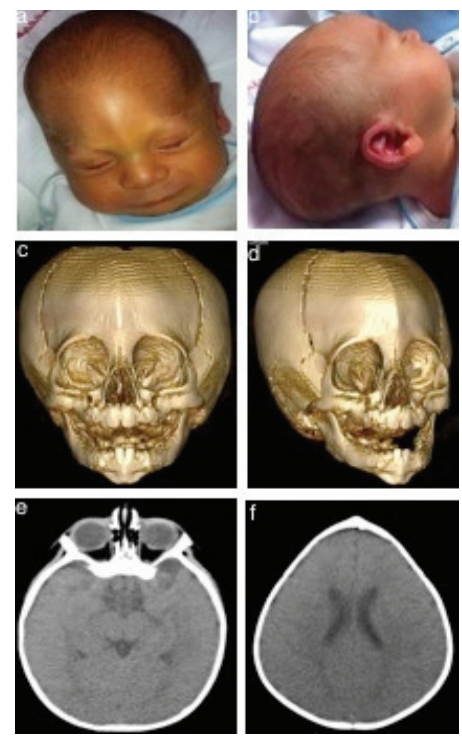

FIGURE 1: Preoperative images. ( $a$ and b) Abnormally shaped skull with hypotelorism; (c- f) fused metopic suture.
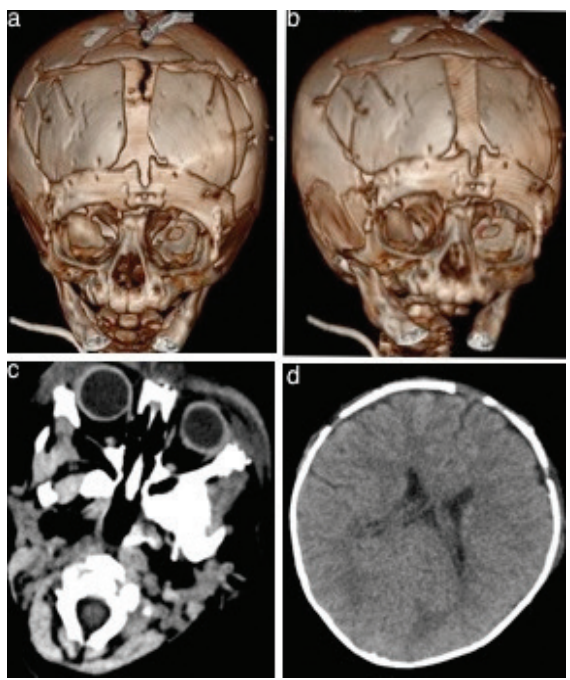

FIGURE 2: Early postoperative images.

The supraorbital rim had fused to form a triangular shape. Using a drill, the supraorbital rim was cut in half and then fused using absorbable plaques leaving $5 \mathrm{~mm}$ apart correcting the triangular shape by aligning the rims on a horizontal plane. The frontal bone was also cut in half, flipped $180^{\circ}$, and then sutured to the parietal and supraorbital bones to ensure anterior cranial widening. The scalp was closed with a pericranial flap in its original position. Postoperatively the patient was monitored in the intensive care
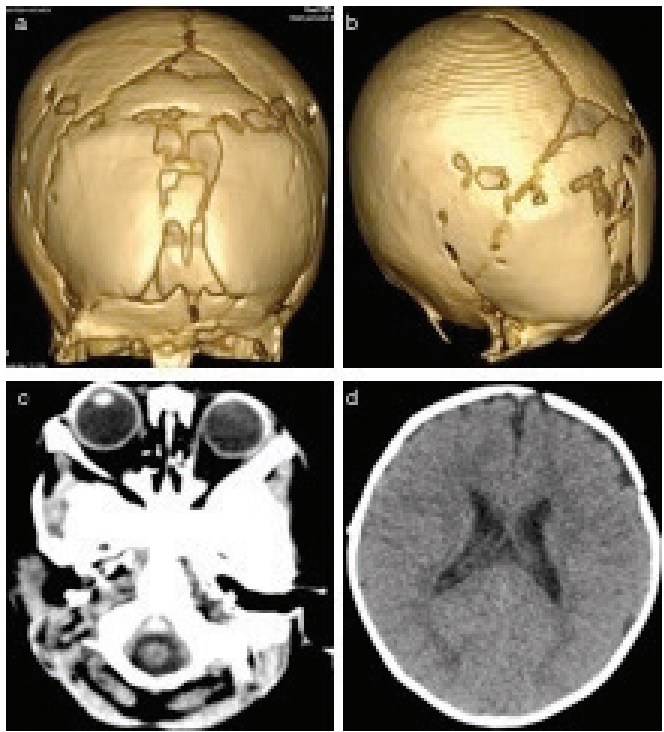

FIGURE 3: Postoperative images at 6 months of age.
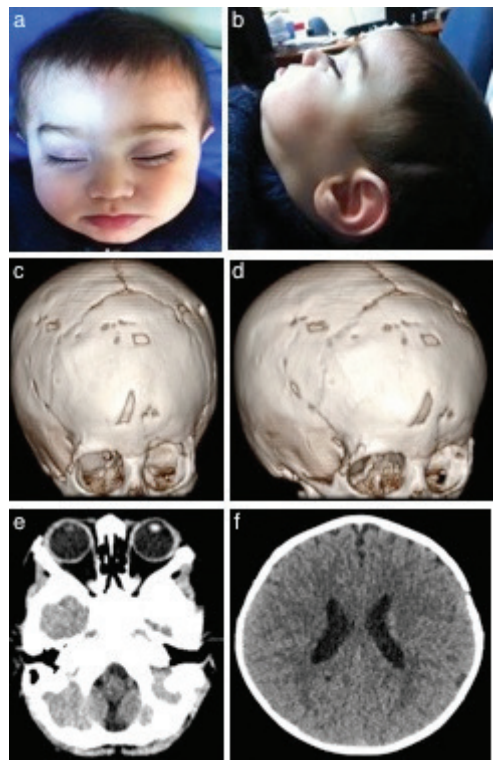

FIGURE 4: First-year postoperative pictures ( $a$ and $b$ ) and images $(c-f)$.

unit and discharged 3 days later with no complications. Early computerized cranial tomography showed no extra complications (Figure 2). Six months and 1 year later he was followed up with a control computerized cranial tomography (Figures 3 and 4).

\section{DISCUSSION}

Surgical techniques for craniosynostosis have evolved over time with Mehner proposing the removal of the suture in 
1921 up to Paul Tessier in 1967, a plastic surgeon who shaped the modern operation techniques with a different perspective. Tessier proposed multiple techniques of osteotomy, bone grafting, and direct correction of deformities, which shaped the craniofacial surgery practiced today $(7,8)$. A wide range of surgical techniques are used for metopic synostosis. This case is an example of fronto-supraorbital advancement, correction of triangular supraorbital rim, and calvarial remodeling.

The incidence of metopic synostosis varies from 1:700 to 1:15,000 newborns with a male to female ratio of 3.3:1 (5). It can be easily diagnosed with direct visualization of the triangularshaped forehead along with palpitation of the probable fused suture. A plain x-ray has lost its value compared with CT, which may reveal a more direct visualization of the fused suture. A three-dimensional reconstruction of the cranial $C T$ is more than diagnostic for the surgeon. Visualization of abnormally closed eyes, hypotelorism, supports the diagnosis. A CT can readily show the cranial contours, a possible hydrocephalus, and the expansion of frontal subarachnoid distance. Cranial magnetic resonance imaging is usually indicated so that a surgeon does not oversee a microcephalic patient, which avoids the need for an operation. If necessary, a technetium-enhanced bone scintigraphy normally reveals low uptake in the newborn, whereas a completely closed suture has no uptake and premature closing reveals a high uptake due to active turnover. Although rare, an urgent surgery is usually indicated in the case of aberrant craniofacial anomaly or a syndrome of increased intracranial pressure. Otherwise, an elective surgery planned before 6 months of age is plausible (9). Traditional surgical technique involves suturectomy of the early fused metopic suture. However, this technique has lost its place in the treatment modalities because the craniectomy defect usually fuses before desired cranial volume along with preservation of hypotelorism, since it does not indicate for the removal and correction of the triangular-shaped supraorbital rims. Each surgical technique aims to correct trigonocephaly while preserving normal cranial volume and reconstructing calvarium most similar to its anatomic plane. The technique mentioned here allows the correction of hypotelorism, cosmetic reconstruction of the calvarium, and preservation of normal cranial volume. In To allow the brain to reach its maximum volume, temporal osteotomies must be performed, but this creates a cosmetic problem of temporal hollowing in the longterm follow-up.

Another common problem faced when dealing with metopic suture synostosis is the correct reshaping of the frontal bandeau. Careful preoperative planning suited for each case requires the surgeon to calculate whether the desired symmetrical and satisfying supraorbital rim contours will be achieved. Frontoorbital rim advancement is the most appropriate technique to achieve the correction of hypotelorism and bifrontal narrowing. Common intraoperative complication of craniosynostosis surgeries is hemodynamic instability of the patient due to blood loss. On average $42 \pm 12.0 \%$ EBV blood loss was recorded for the surgical correction of metopic synostosis (10). However, technological improvements, especially with plaques and screws, have decreased surgical time, thus decreasing the amount of blood loss and necessity for transfusion.

\section{CONCLUSIONS}

Fronto-orbital advancement and remodeling is an effective technique to correct metopic suture synostosis. However, a surgeon must keep in mind that each case of trigonocephaly requires a unique approach with additional maneuvers such as total calvarial remodeling and the use of absorbable plaques. To treat hypotelorism, fronto-orbital rim advancement is a must. This modality results in improved cosmetic skull form with restored cranial volume, hence leading to higher patient and parent satisfaction.

\section{REFERENCES}

1. Di Rocco F, Arnaud E, Renier D. Evolution in the frequency of nonsyndromic craniosynostosis: Clinical article. Journal of Neurosurgery: Pediatrics. 2009;4(1):21-5.

2. Governale LS. Craniosynostosis. Pediatr Neurol. 2015;53(5):394401.

3. Vu HL, Panchal J, Parker EE, Levine NS, Francel P. The timing of physiologic closure of the metopic suture: a review of 159 patients using reconstructed 3D CT scans of the craniofacial region. Journal of Craniofacial Surgery. 2001;12(6):527-32. 
4. Weinzweig J, Kirschner RE, Farley A, Reiss P, Hunter J, Whitaker LA, et al. Metopic synostosis: Defining the temporal sequence of normal suture fusion and differentiating it from synostosis on the basis of computed tomography images. Plastic and reconstructive surgery. 2003;112(5):1211-8.

5. Lajeunie E, Le Merrer M, Marchac D, Renier D. Syndromal and nonsyndromal primary trigonocephaly: analysis of a series of 237 patients. Am J Med Genet. 1998;75(2):211-5.

6. Alderman BW, Fernbach SK, Greene C, Mangione EJ, Ferguson SW. Diagnostic practice and the estimated prevalence of craniosynostosis in Colorado. Arch Pediatr Adolesc Med. 1997;151(2):159-64.
7. van der Meulen J. Metopic synostosis. Childs Nerv Syst. 2012;28(9):1359-67.

8. Ghali MG, Srinivasan VM, Jea A, Lam S. Craniosynostosis surgery: the legacy of Paul Tessier: Historical vignette. Neurosurgical focus. 2014;36(4):E17.

9. Hormozi AK, Shahverdiani R, Mohammadi HR, Zali A, Mofrad HR. Surgical treatment of metopic synostosis. J Craniofac Surg. 2011;22(1):261-5.

10. Kearney RA, Rosales JK, Howes WJ. Craniosynostosis: an assessment of blood loss and transfusion practices. Can J Anaesth. 1989;36(4):473-7. 